\title{
Adherence to malaria rapid diagnostic test results and a qualitative study on Health Workers perception about the test in Agona East District of Ghana
}

Selina Ansah-Koi ( $\sim$ maakobe@gmail.com )

University of Ghana

\section{Research}

Keywords: Rapid Diagnostic Test (RDT), Community Health and Planning Services (CHPS), Health Center, Malaria and Adherence

Posted Date: December 2nd, 2020

DOl: https://doi.org/10.21203/rs.3.rs-118883/v1

License: (9) (1) This work is licensed under a Creative Commons Attribution 4.0 International License. Read Full License 


\section{Abstract}

Background Ghana has adopted the policy of diagnostically testing for malaria before treatment. The Rapid Diagnostic Test (RDT) is used to diagnose malaria especially in lower health facilities like Health Centers (HC) and Community-based Health Planning Services (CHPS) Compounds without microscopy. Studies have shown that clinicians do not always adhere to negative malaria test results of the RDT and prescribe anti-malarials.

Methods Quantitative aspect focused on how malaria is diagnosed and adherence to negative rapid diagnostic test results using the consulting room register and patients' folder of six health facilities within the period of July to September 2015.

In-depth interviews using purposive sampling was conducted for 14 health workers at Health Centers and CHPS compounds to find out their perceptions about malaria RDT and reasons for adherence or non-adherence to test results.

Results Of the six health facilities in the Agona East District, 3.87\% (14/362) were presumptively diagnosed with malaria whiles $96.10 \%$ (348/362) were diagnosed with malaria using RDT.

For those tested with RDT, $15.25 \%$ tested negative and $84.75 \%$ tested positive. Of those who tested negative, $20.75 \%$ were given anti-malarials and $79.25 \%$ did not receive anti-malarials. Health workers perceived the Malaria RDT kit as easy to use and also as making work easier. Testing is done mainly because it is a policy.

Conclusion The malaria testing rate in the Agona East District is higher than that at the National level. Health Centers compared to CHPS compounds are likely to treat for malaria in cases of a negative test result. There is also high adherence to negative test results. Health workers perceive the RDT as a useful tool which has made malaria diagnosis easy.

\section{Background}

In spite of the various interventions, a million deaths and more than 400 million cases yearly are as a result of malaria. Out of these deaths, $90 \%$ happen in Sub Saharan Africa (Bilal, 2015). Ghana has a high incidence of malaria and everyone in the country is at risk of being infected with malaria (Fenny et al., 2014).

The main approaches to preventing and controlling malaria are early detection and early treatment (Abreha et al., 2014). To reduce malaria mortality, several interventions have been introduced. They include the introduction of Artemisinin-based Combination Therapy (ACT), the use of insecticide-treated bed nets and Rapid Diagnostic Test. These interventions have in a way contributed to a reduction in the number of malaria cases(Stresman et al., 2012).

Policymakers including the WHO for many years promoted the idea of presumptively diagnosing for malaria and use of chloroquine for treatment of suspected malaria cases. The main reason being chloroquine is cheap and accepted by many (Ochodo et al., 2016b)

Presumptively diagnosing malaria though not always accurate is still the basis for therapeutic care in areas without laboratory facilities or where malaria tests are not needed. This can result in wastage of drugs, and an increase in resistance and missed cases of malaria (Eibach et al., 2013a). 
The WHO in 2012 launched an initiative which focuses on testing, treating and tracking to address the need of ensuring anti-malarials are given based on results as well as increase reporting cases (Eibach et al., 2013b).

There are 44 malaria-endemic countries under the WHO Africa region. As at 2012, 41 out of this figure had adopted the policy of testing before treatment of malaria for all age groups (Bastiaens et al., 2014). The Ministry of Health, Ghana in 2004 adopted the policy of testing for malaria before treatment in line with WHO directives this was to ensure anti-malarials are given to patients who need it and also reduce over diagnosis and misdiagnosis of malaria (Rauf et al., 2014). According to the 2014 Guideline for case management of malaria in Ghana, diagnosing malaria must be done with either microscopy or rapid diagnostic test irrespective of age group. Diagnosis based on symptoms must only be done in the absence of microscopy and rdt.

Between 2005 to 2007, the number of suspected malaria cases that received treatment was 18\% and this remained the same however in 2012 it increased to 35\% (NMCP, 2013). The number of OPD cases that were tested before treatment in Ghana was 42.9\% in 2011 and this increased in 2014 to 74.3\% (Ghana Health Service, 2015)

Microscopy is the gold standard for malaria diagnosis (Diakite et al., 2012). Aside microscopy, the WHO has accepted the use of malaria rapid diagnostic test for malaria diagnosis in lower health care(Asiimwe et al., 2012). The Malaria Rapid Diagnostic Test can detect antigens of malaria parasites within a time frame of 10-15 minutes. It is used by pricking a finger to obtain drops of blood which is then put on a test strip (Zongo et al., 2016)

One does not need to be skilled to use RDT and usage is not difficult. It can be used for malaria detection in lower level health facilities where microscopy is not available (Kyabayinze e $t$ al, 2012). RDTs when used in field conditions, helps not only in detecting malaria among people with fever but also helps those who test positive get prompt treatment (Pan, 2015). The protocol for the management of malaria in Ghana recommends that higher health facilities like hospitals and health centers use microscopy while peripheral facilities like CHPS compounds use RDT (Fenny et al., 2014). According to the guidelines for case management of malaria in Ghana 2014, patients must not be treated with anti-malarials when they test negative for malaria, rather investigation needs to be done to find out the cause of illness.

Numerous published studies have shown that once rdts are introduced in areas relying on symptoms for malaria diagnosis, there is a sharp decrease in anti-malaria prescriptions (Bastiaens et al., 2014).

Before Ghana adopted the policy of test before treatment, $45 \%$ of those who tested negative for malaria were still prescribed with anti-malarials (Boadu et al., 2016).

Another study done on public health facilities in a rural district in Ghana revealed that in areas already using microscopy for malaria detection, RDTs introduction had no impact on prescribing practices, however, in areas relying on presumptive diagnoses, the introduction of RDT has led to a decrease in anti-malaria overprescription. However, $50 \%$ of those who tested positive still received anti-malarial treatment (Chandler et al., 2010).

Adherence to results is an important factor in deciding whether RDT can reduce cost and improve the management of malaria. Not adhering to results is therefore a waste of resources and time (Bisoffi et al., 2009) 
In areas where traditionally fever is treated as malaria, a negative RDT test may lead to doubt most especially if the community members have no trust in the new testing format as well as in modern medicine (Miller \& Sikes, 2015). Studies that have looked at perceptions of patients, caregivers and clinicians in relation to malaria diagnosis has revealed two major pictures: the first is that a patient would want to receive treatment for malaria after being tested, the second is that results of the test may not have much influence on clinicians prescription practices (Derua et al., 2011)

This study assessed how malaria is diagnosed in lower health facilities in the Agona East District and adherence to test result. It also looked at perceptions health workers have about the Rapid Diagnostic Test.

\section{Methods}

\section{Study Area}

The study was conducted in the Agona East District of the Central Region of Ghana. According to the Population and Housing Census 2012, the area has a population of 85,920 inhabitants. The area is divided into five administrative sub-districts. These are Kwanyako, Nsaba/Duakwa, Asafo, Mankrong and Mensakrom.

The area has three private maternity homes, eleven CHPS compounds, five governmental health centers and one health center under the Christian Health Association of Ghana.

The entire District has only one governmental health facility which uses microscopy for malaria detection and it is situated at Kwanyako. All the others use RDT for malaria detection.

\section{Study Design}

The study was a cross-sectional study using both quantitative and qualitative methods. Quantitative method was used to find out how malaria is diagnosed and adherence to negative test results. The qualitative method looked at reasons for adherence or non-adherence to negative test results and perceptions health workers have about RDT.

\section{Quantitative Method}

To find out how malaria was diagnosed, there was a review of the consulting room register and patients' folders within July and September 2015 for six randomly selected health facilities comprising of three health centers and three CHPS compounds. Inclusion criteria were governmental health facilities that use rdt and not microscopy for malaria detection.

The sample size was calculated using Cochran's formula $n=Z^{2} p q / e^{2}$

Based on the formula, $\mathrm{n}$ is the sample size, $\mathrm{z}$ is the confidence interval of $95 \%$ (standard value of 1.96), e is the margin of error of $5 \%$ (standard value of 0.05 ) and $\mathrm{P}$ is $30 \%(0.30)$. This is the estimated number of malaria cases nationally. According to the Ghana Health Service 2014 Annual Report, malaria accounts for about $30 \%$ of all outpatient cases in Ghana as at 2014.

$q$ is 1-p which is 0.7 
Therefore the sample size was calculated using Cochran's formula as follows:

$\mathrm{n}=1.96^{2}(0.30 * 0.7) / 0.05^{2}$

$\mathrm{n}=3.8416 * 0.21 / 0.0025$

$\mathrm{n}=322.7$ or 323

Thus the minimum sample size of 323 .

The total sample size used for the study was 362 . That for the three CHPS compound was 116 and that of the three Health Centers was 246.

\section{Sampling Procedure for the Health Centers}

The district has a total of five governmental health centers, one for each sub-district. One facility was excluded because microscopes are used for malaria detection and the study focused on facilities using only RDT. Three out of the remaining four were randomly selected. Systematic sampling was used to get data for each of the facilities visited.

Systemic sampling was done using the formula $k=N / n$ was used to get the data for each facility.

From the formula, $\mathrm{k}$ is the sampling interval, $\mathrm{N}$ is the population size and $\mathrm{n}$ is the sample size

The consultation room register was used to find out the total provisional malaria cases for the periods of July to September 2015 to get the sampling frame. The figure obtained was divided by the expected sample size of each facility which was 86 . The figure obtained after division served as the interval for the selection of cases.

In cases where the person tested negative, a request was made for the folder to find out if anti-malarials were prescribed, symptoms of the patient and temperature.

A total of 246 was obtained from the three health centers. This represented the total malaria diagnoses.

Data was collected and entered into Microsoft excel. Data was then imported into STATA 13.1 for analysis.

\section{Sampling Procedure for CHPS compounds}

There are eleven CHPS compounds out of which three were randomly sampled, one for each sub-district. The consulting room register was assessed for the total number of patients provisionally diagnosed with malaria within July-September, 2015. Since CHPS compounds have fewer cases compared to health centers, the total number of provisional malaria cases for the period was what was recorded. After obtaining this figure, checks were done to determine those that were tested before treatment and those that were presumptively diagnosed and treated for malaria.

The total malaria cases for the period for facility 1 was 23 , facility 2 was 33 and facility 3 was 60 . This gave a total of 116 .

\section{Qualitative Method}


Qualitative data was aimed at exploring reasons for adherence or non-adherence to malaria test results as well as find out perceptions health workers have about RDT. This was done using in-depth interviews with different cadres of health workers such as a physician assistant, enrolled nurses, community health officers and community health workers.

Purposive sampling was used to select health workers who can diagnose and treat malaria within government health centers and CHPS compounds in the Agona East District. To ensure fair representation, participants were selected from all the four health centers and five CHPS compounds including facilities whose records were accessed for the quantitative aspect of the research. Interviews were conducted until a point where no new information was being obtained.

A total of fourteen in-depth interviews were done. Interviews were audio recorded and fully transcribed into a word processing document. Transcribed data underwent content analysis by site and based on themes in the interview guide.

\section{Results}

\section{Quantitative Results}

\section{Proportion Diagnosed with malaria presumptively compared to the proportion tested before treatment}

From table 1, 2.44\% (6/246) people were diagnosed and treated for malaria without testing and $97.56 \%$ $(240 / 246)$ were tested for malaria before treatment for the three health centers.

For the three CHPS Compounds, 6.90\% (8/116) were presumptively diagnosed for malaria whiles $93.10 \%$ $(108 / 116)$ were tested for malaria using RDT.

Table 1 Type of malaria diagnosis for Health Facilities

\begin{tabular}{|c|c|c|c|c|}
\hline & Presumptive Diagnosis N(\%) & Tested with RDT & $\mathrm{N}(\%)$ & Total N(\%) \\
\hline Health Centers & $6(2.44)$ & $240(97.56)$ & & $246(100)$ \\
\hline CHPS Compounds & $8(6.90)$ & $108(93.10)$ & & $116(100)$ \\
\hline Total & $14(3.87)$ & $348(96.13)$ & & $362(100)$ \\
\hline
\end{tabular}

\section{Results for those tested with MRDT}

Out of those tested with the RDT, 83.75\% (201/240) tested positive for the health centers whiles $16.25 \%$ (39/240) tested negative. For the CHPS compound, 87.04\% (94/108) tested positive and 12.96\% (14/108) tested negative (Table 2).

Table 2 Results of those tested with RDT 


\begin{tabular}{|c|c|c|c|c|c|}
\hline & RDT Positive & $\mathbf{N}(\%)$ & RDT Negative & $N(\%)$ & Total N(\%) \\
\hline Health Centers & $201(83.75)$ & & $39(16.25)$ & & $240(100)$ \\
\hline CHPS Compounds & $94(87.04)$ & & 14(12.96) & & $108(100)$ \\
\hline Total & $295(84.77)$ & & $53(15.23)$ & & $348(100)$ \\
\hline
\end{tabular}

\section{Adherence to RDT negative results}

For the three health centers, $25.64 \%$ (10/39) received malaria treatment in spite of a negative test result whiles $74.36 \%$ (29/39) were not treated for malaria. In the case of the CHPS Compounds, 7.14\% (1/14) were treated for malaria although the result was negative and $92.86 \%(13 / 14)$ were not treated for malaria (Table 3).

Thus for the total Health centers and CHPS compound, anti-malarials were given to $20.75 \%(11 / 53)$ of the patients who had a negative malaria test result and $79.25 \%(42 / 53)$ patients who tested negative were not treated for malaria.

Table 3 Adherence to RDT negative results

\begin{tabular}{|c|c|c|c|}
\hline & Given anti-malarial $\mathrm{N}(\%)$ & Not given anti-malarial $\mathbf{N}(\%)$ & Total N(\%) \\
\hline Health Centers & $10(25.64)$ & $29(74.36)$ & $39(100)$ \\
\hline CHPS Compounds & $1(7.14)$ & $13(92.86)$ & $14(100)$ \\
\hline Total & $11(20.75)$ & $42(79.25)$ & $53(100)$ \\
\hline
\end{tabular}

\section{Qualitative Results}

Table 4 Demographic details of IDI participants 


\begin{tabular}{ll}
\hline Variable & N=14 (n\%) \\
\hline Type of facility participants works with & \\
Health Center & $9(35.71)$ \\
CHPS Compound & $5(64.29)$ \\
Cadre of health workers & \\
$\quad$ Community Health Nurse & $1(7.14)$ \\
$\quad$ Community Health Officer & $1(7.14)$ \\
$\quad$ Enrolled Nurse & $5(35.71)$ \\
$\quad$ Physician Assistant & $1(7.14)$ \\
$\quad$ Principal Midwifery Officer & $1(7.14)$ \\
Senior Midwifery Officer & $1(7.14)$ \\
Senior Staff Nurse & $1(7.14)$ \\
Staff Community Health Nurse & $1(7.14)$ \\
Staff Nurse & $2(14.29)$ \\
\hline Sex & \\
Female & $9(64.29)$ \\
Male & $5(35.71)$ \\
\hline Duration Working At Current Position & \\
1 year & $2(14.29)$ \\
2 years & $3(21.43)$ \\
4 years & $2(14.29)$ \\
Years & $4(28.57)$ \\
\hline Whether trained in the use of RDT & $2(14.29)$ \\
\hline & $1(7.14)$ \\
\hline
\end{tabular}

\section{Perceptions about RDT and adherence among health workers}

Perceptions identified after the in-depth interviews were grouped into four thematic areas. Perception: logic of testing, perception: RDT, perception: early detection of the malaria parasite and reasons for adherence or nonadherence to test results.

Perception: Logic of testing 
Most of the people interviewed were of the view that the main logic for testing with RDT was in compliance with the national policy which requires testing before treatment. Most of the staff at the CHPS Compounds confirmed this. The following quotes illustrate that:

"It is a guideline we are following, the policy says test before treating so, so far as the client has come and shown the symptoms you have to test"[CHN, CHPS compound]

"The policy is saying you should test and treat. So far as the test has shown to be negative, you will not treat for malaria but other conditions and we have tried and it is getting us good results" [CHO, CHPS compound]

It is worth noting that the symptoms presented by the patients guided the use of RDT. Symptoms such as fever, headache, chills, vomiting and diarrhoea. A community nurse made the statement below:

"If the sign shows to be malaria then we test for malaria with the RDT before we can treat. There are some cases like someone comes with a wound and doesn't show any sign of malaria so we don't test"[Enrolled nurse, HC]

Nevertheless, there was the tendency to test for malaria although patients show no symptoms. The quote below shows that:

"Actually where we are, we know our cases are more or less malaria, we are in a malaria prone zone...sometimes when they come in with wound, we try to do the RDT and sometimes it comes out positive. So any case that comes we try to do the RDT not only when you are complaining of malaria symptoms"[CHO, CHPS compound]

\section{Perception: RDT}

All the health workers interviewed agreed that the introduction of RDT had made their work easy. Once the person shows symptoms of malaria all you need to do is test and treat accordingly. The following quotations sum it up:

"It has been helping us a lot because we don't have a medical assistant or doctor around so it helps us with the diagnosis"[Enrolled Nurse, HC]

"RDT is very very good because it makes the work very easy for you, when the person comes and you suspect malaria when you check, when it's positive you just treat the person and you are free, you don't have to probe and probe and probe whether it's malaria or not". [CHN, CHPS compound]

"It's helping us pinpoint the malaria cases and it helps us give the right treatment to the patient" [Enrolled Nurse, $H C]$

"It has helped us as a whole as a health service to realise that the number of malaria cases we were diagnosing at first were not actually all malaria and so now the rate at which the disease is occurring is coming down a bit" [Physician Assistant, HC]

Not only has the introduction of RDT made their work easy but has also increased their confidence in treating malaria. One community health worker in a remote area admitted that using RDT distinguishes them from quack doctors and increases the confidence patients have in them. 
"It has also helped show we are professionals. Sometimes I go for home visits, those quack doctors don't test before treating so when they see am testing, they say no, this is the right thing am doing"[CHO, CHPS

compound]

\section{Perception: early detection of malaria parasites}

Personnel interviewed admitted there are instances where the kit would not be able to identify the malaria parasite.

"This RDT detects only the falciparum but we have other types of malaria so perhaps the person is showing the other types" [staff community health nurse, CHPS compound]

"We have been having the notion that at times it doesn't pick the malaria parasites so I don't know if that is true, there are times when a person presents symptoms of malaria and you test and it is negative so I tell you at times this kit doesn't pick so go to the lab, I don't know if it is the right thing but that is what we do" [Enrolled nurse, HC].

"We were made to know that at early stages sometimes you can check and the sample that you pick, it will not contain the parasite so it will read negative so at that stage you don't treat or give anti-malarials. [CHO, CHPS compound]

Health workers from both Health Centers and CHPS compounds cited cases where patients test negative and retest after a few days shows a positive result. In some instances, the results will be negative but turn out to be positive after they are referred to go for microscopy.

"...me myself I have experienced that before. I used the RDT twice but it was negative but when I went to the lab and they did the microscopy it was plus plus" [CHO, CHPS compound]

Another reason why an RDT test may not detect the parasite is patients take in anti-malarials drugs before coming to the facility

"Some people will be taking drugs before coming...so once you test it will be negative"

\section{Reasons Accounting for adherence and non-adherence to RDT results}

Confidence in RDT results and constraints: what to do were the two broad themes identified regarding factors accounting for adherence and non-adherence to results.

\section{Confidence in RDT results}

All personnel interviewed had confidence in a positive test result and treated for malaria. However, in cases of a negative test result, there were mixed responses regarding treatment for malaria especially in instances where the patients showed signs and symptoms of malaria.

The quotations below show the confidence they have with test results. 
"when you come with complains of malaria, and I test for malaria and it is positive that is when I have confidence but at times they show signs and you test and it is negative, and if you go to the laboratory, you test and it is positive". [Enrolled nurse, $\mathrm{HC}$ ]

"...sometimes we don't believe (the results) that it why we refer the person" [Principal midwifery officer, HC]

Human factors were identified as possible causes of false-negative test results. Factors such as the amount of blood and buffer used, not waiting long enough to read the results were identified by the respondent. This was summed up by a Physician Assistant by saying

"I wouldn't say a challenge with the kit, I would say probably with the people who do it, with the volume of blood they have to take, the volume of the buffer they have to add. Those ones affect it that much. And then sometimes too how people read is also another challenge. The line might be somehow faint so if they can't see, they just write negative there. I think those are the major challenges I've found". [Physician assistant, HC]

A midwife at a health Center supported the above view and said:

"There are times we are in a hurry so that initially we thought it is negative but afterwards when the result is left for a while, about twenty minutes we see that it becomes positive. It is the timing."

Generally, all agreed the RDT is beneficial but an enrolled nurse was of the view that it also affects the perception people have about the health facility. There are instances where clients are referred to higher facilities or other health centers especially in cases where the test turns out negative although the patients show symptoms of malaria. Below is what she said

"it doesn't make our work that effective, because when they come you have to refer, when they come you have to refer and with this, you will see, they will lose confidence in us"[Enrolled nurse, HC]

\section{Constrain what to do:}

The health workers had divergent views regarding what to do once a suspected case of malaria tests negative. In such instances, medication is given to patients to treat underlying conditions and patients are asked to go home and come after some days for retesting or once the condition worsens. Other alternatives include referring the patient to a higher health facility or treating the patient for malaria.

The quotes below shows what is done in such instances.

"when someone comes and is complaining of vomiting we check and see if it is RDT positive. If it is positive we treat if not then we have to treat only vomiting"[Enrolled nurse, $\mathrm{HC}]$

"you tell the client that you know this early stage it couldn't pick the parasite so it will read negative so you manage the signs and symptoms so that she goes and later when she feels other complication or when she's having more symptoms she should come for retesting" [CHN, CHPS compound]

Workers at the health centers generally said patients with symptoms of malaria but negative test results are referred to higher health facilities for microscopy testing. 
CHPS compound staff do not have much confidence in a negative test result however they prefer to do a retest after some days before treatment. In cases where treatment is given, they need to consult a health staff before treatment for malaria.

"If it is negative, I will have to call the medical assistant so she will give me the guidelines whether I should treat or I should do other diagnosis or ask further questions"[CHO, CHPS compound]

In instances where the patients shows symptoms of malaria but test negative and further probing does not help with identifying the underlying condition, the patients is treated for malaria. The quotes below confirms that.

"The client will come maybe fever very high and then will be presenting with chills and sometimes with night sweat, headache and then probably some of them will even be having some kind of psychosis and then you do the RDT and then RDT is negative and then as a clinician you try asking so many questions to rule out any other disease conditions and you find out that you are not getting any positive result and so you just have to go ahead and treat."[Physician Assistant, HC]

\section{Discussion}

Malaria can be diagnosed either presumptively or by testing. Presumptive diagnosis is usually done in areas without laboratory facilities or skilled personnel to conduct the test. Health centers and CHPS compounds are lower health facilities in Ghana that rely on RDT usage. There are various types of tests to diagnose malaria among which includes the rapid diagnostic test.

Diagnosing malaria based on symptoms can result in anti-malarial drug abuse which can lead to resistant strains of the parasite, missed cases of malaria and misuse of resources (Frank Baiden et al, 2012). From the study, $96.13 \%$ of cases were tested for malaria for the six health facilities in the Agona East district and $3.87 \%$ were diagnosed presumptively. This figure compared to the National Testing rate as at 2014 is higher. In Ghana, as at 2014, 74.3\% of OPD cases were tested for malaria before receiving treatment (Ghana Health Service 2014 Annual Report). The testing rate in the Agona East District is also higher compared to that of the African subregion which is 64\% (Ochodo et al., 2016a). Studies conducted in different areas in Africa and Asia confirms that RDT has a substantial advantage over presumptive diagnosis and when used in field conditions works just as light microscopy or even better (Hansen et al., 2015). Using symptoms for the diagnosis of malaria is done in many areas in Africa although this method comes with problems (Ansah et al., 2013). Malaria when under diagnosed can result in treatment delay and can even progress to death (Derua et al., 2011).

From the qualitative aspect of the study, health workers agreed that RDT was used because the protocol required that. Symptoms such as fever and vomiting require that patients are tested.

It is worth noting that some facilities tested patients for malaria irrespective of the symptoms presented. A health worker during the interview said this was done because the area is malaria endemic.

From the qualitative aspect of the study, the health workers agreed that the RDT was a useful working tool and this shows a likely endorsement of testing over symptomatic diagnosis.

\section{Treatment for negative cases}

Page $12 / 17$ 
In cases where the patient shows symptoms of malaria but tests negative, health workers resorted to one of these measures. They refer the patient to a facility with microscopy for further testing, gave medication for the symptoms and asked patients to come for retesting after some days. Another option is providing antimalarial treatment.

From the study, a total of $20.75 \%(11 / 53)$ of patients who tested negative for malaria, were still treated for malaria. Out of this figure, $7.14 \%$ (1/14) were from CHPS compounds and $25.64 \%(10 / 39)$ from health centers. These figures are lower compared to a study by Chandeller et al (2010) in rural facilities in Ghana where $45.5 \%$ of patients who tested negative for malaria were still treated for malaria. A reduction in malaria over prescription can imply experience, acceptance, and appropriate training in the use of RDT (Mubi et al., 2013). Studies conducted in Zambia and Tanzania showed a decrease in ACT prescriptions by Community Health Workers using RDT (Mukanga et al., 2012). A study of rural facilities in Ghana showed that $45.5 \%$ of patients who tested negative were given anti-malarials(Chandler et al., 2010).

Further study on local health workers in Uganda showed that $92 \%$ of clinicians saw a positive RDT as prove of malaria infection however $49 \%$ viewed a negative test result was enough evidence to rule out malaria(Miller \& Sikes, 2015).

When all fevers are treated as malaria, certain serious conditions will be masked (Fenny et al., 2014) therefore adhering to test results is vital.

A study showed clinicians are reluctant to prevent treatment for malaria in negative cases. Adherence to test results is important in assessing if RDT is useful in malaria management (Bisoffi et al., 2009).

Lack of training, minimal supervision, socio-cultural aspects and lack of trust in the testing system were factors identified as accounting for poor adherence to the guideline of malaria treatment in Africa (Salomão et al., 2015)

\section{Perception}

A study by Asiime et al (2013) on the perception of health workers on RDT in Uganda saw RDTs as an empowerment tool for health workers and they had confidence in a positive result compared to presumptive diagnosis.

From the study, human factors such as inadequate buffer and blood volume as well as hurriedly reading RDT results were identified as factors affecting RDT results. A study on the behaviour of health workers in Southeastern Nigeria by Uzocukwu et al (2013) revealed that inadequate blood volume and not reading results on time reduced the test's sensitivity. The quality of the rapid diagnostic test can be affected by factors such as transport, storage, manufacturing standard and end-user use of the kit (Moonasar et al., 2009).

\section{Conclusion}

From the results, the testing rate in the Agona East district is higher compared to that at the national level. Also, the proportion of patients who tested negative but still receive treatment for malaria was on the low side with health centers much more likely to treat negative cases as compared to CHPS compounds. Based on the 
interviews with health workers it was obvious they had confidence in a positive test result however there was little confidence in negative malaria rdt especially in cases where the patient shows symptoms of malaria.

Giving malaria treatment in spite of a negative test result can mask other underlying conditions which can be fatal. Asking the patients to go home and return when the condition worsens can also be fatal. Numerous cases where cited whereby client tested negative however referrals for microscopy testing came out positive. There is therefore the need to get RDT kits with high sensitivity and specificity to increase confidence in negative test results.

\section{List Of Abbreviations}

CHN Community Health Nurse

$\mathrm{CHO} \quad$ Community Health Officer

CHPS Community-based Health Planning Services

EN Enrolled Nurse

HC Health Center

RDT Rapid Diagnostic Test

WHO World Health Organization

\section{Declarations}

\section{Ethics approval and consent to participate}

Ethical clearance for the study was obtained from the Ethical Review Committee of the Ghana Health Service. Ethics Review Committee number GHS-ERC 80/02/16. Consent was sought from the District Health Directorate to assess the health records of selected health facilities in the Agona East district. Participants who took part in the study filled an informed consent form.

\section{Consent for publication}

Participants who took part in the interview filled an informed consent form.

\section{Availability of data and material}

The datasets used and analyzed for the study are available from the author on request.

\section{Competing interests}

The author declares there are no competing interests.

\section{Funding}


This study was funded by TDR, the Special Programme for Research and Training in Tropical Diseases, which is hosted at the World Health Organization and co-sponsored by UNICEF, UNDP, the World Bank and WHO by the World Health Organization/ Tropical Disease Research (WHO/TDR). Grant number B40300.

\section{Acknowledgement}

Not applicable

\section{Authors information}

The author has a Masters Degree in Public Health for the University of Ghana, Legon

\section{References}

Abreha, T., Alemayehu, B., Tadesse, Y., Gebresillassie, S., Tadesse, A., Demeke, L., Zewde, F., Habtamu, M., Tadesse, M., Yadeta, D., Teshome, D., Mekasha, A., Gobena, K., Bogale, H., Melaku, Z., Reithinger, R., \& Teka, H. (2014). Malaria diagnostic capacity in health facilities in Ethiopia. 1-8.

Ansah, E. K., Epokor, M., Whitty, C. J. M., Yeung, S., \& Hansen, K. S. (2013). Cost-Effectiveness Analysis of Introducing RDTs for Malaria Diagnosis as Compared to Microscopy and Presumptive Diagnosis in Central and Peripheral Public Health Facilities in Ghana. 89(4), 724-736. https://doi.org/10.4269/ajtmh.13-0033

Asiimwe, C., Kyabayinze, D. J., Kyalisiima, Z., Nabakooza, J., Bajabaite, M., Counihan, H., \& Tibenderana, J. K. (2012). Early experiences on the feasibility , acceptability , and use of malaria rapid diagnostic tests at peripheral health centres in Uganda-insights into some barriers and facilitators. Implementation Science, 7(1), 5. https://doi.org/10.1186/1748-5908-7-5

Bastiaens, G. J. H., Bousema, T., \& Leslie, T. (2014). Scale-up of Malaria Rapid Diagnostic Tests and ArtemisininBased Combination Therapy: Challenges and Perspectives in Sub-Saharan Africa. 11(1), 1-4. https://doi.org/10.1371/journal.pmed.1001590

Bilal, J. A. (2015). Poor adherence to the malaria management protocol among health workers attending underfive year old febrile children at Omdurman Hospital , Sudan. February. https://doi.org/10.1186/s12936-0150575-9

Bisoffi, Z., Gobbi, F., Angheben, A., \& Ende, J. Van Den. (2009). The Role of Rapid Diagnostic Tests in Managing Malaria. 6(4), 6-7. https://doi.org/10.1371/journal.pmed.1000070

Boadu, N. Y., Amuasi, J., Ansong, D., Einsiedel, E., Menon, D., \& Yanow, S. K. (2016). Challenges with implementing malaria rapid diagnostic tests at primary care facilities in a Ghanaian district: a qualitative study. Malaria Journal, 1-12. https://doi.org/10.1186/s12936-016-1174-0

Chandler, C. I. R., Whitty, C. J. M., \& Ansah, E. K. (2010). How can malaria rapid diagnostic tests achieve their potential? A qualitative study of a trial at health facilities in Ghana. 1-15.

Derua, Y. A., Ishengoma, D. R. S., Rwegoshora, R. T., Tenu, F., Massaga, J. J., Mboera, L. E. G., \& Magesa, S. M. (2011). Users ' and health service providers ' perception on quality of laboratory malaria diagnosis in Tanzania. 
$1-7$.

Diakite, M., Diallo, M., Sagara, I., Masinde, G. L., Doumbo, S. N., Dolo, A., ... Krogstad, D. J. (2012). False-Negative Rapid Diagnostic Tests for Malaria and Deletion of the Histidine-Rich Repeat Region of the hrp2 Gene \{, 86(2), 194-198. http://doi.org/10.4269/ajtmh.2012.10-0665

Eibach, D., Traore, B., Bouchrik, M., Coulibaly, B., Coulibaly, N., Siby, F., Bonnot, G., Bienvenu, A., \& Picot, S. (2013a). Evaluation of the malaria rapid diagnostic test VIKIA malaria Ag Pf / Pan ${ }^{T M}$ in endemic and nonendemic settings. 1-7. https://doi.org/10.1186/1475-2875-12-188

Eibach, D., Traore, B., Bouchrik, M., Coulibaly, B., Coulibaly, N., Siby, F., Bonnot, G., Bienvenu, A., \& Picot, S. (2013b). Evaluation of the malaria rapid diagnostic test VIKIA malaria Ag Pf / Pan TM in endemic and nonendemic settings. $1-7$.

Fenny, A. P., Hansen, K. S., Enemark, U., \& Asante, F. A. (2014). Quality of uncomplicated malaria case management in Ghana among insured and uninsured patients. 1-12.

Ghana Health Service. (2015). 2014 Annual Report of the Ghana Health Service. July, 1-135. https://www.ghanahealthservice.org/downloads/Ghana_Health_Service_2014_Annual_Report.pdf

Hansen, K. S., Grieve, E., Mikhail, A., Mayan, I., Mohammed, N., Anwar, M., Baktash, S. H., Drake, T. L., Whitty, C. J. M., Rowland, M. W., \& Leslie, T. J. (2015). Cost-effectiveness of malaria diagnosis using rapid diagnostic tests compared to microscopy or clinical symptoms alone in Afghanistan. ???, 15-17.

https://doi.org/10.1186/s12936-015-0696-1

Kyabayinze, D. J., Asiimwe, C., Nakanjako, D., Nabakooza, J., Bajabaite, M., Strachan, C., ... Geetruyden, J. P. Van. (2012). Programme level implementation of malaria rapid diagnostic tests (RDTs ) use: outcomes and cost of training health workers at lower level health care facilities in Uganda. BMC Public Health, 12(1), 1. http://doi.org/10.1186/1471-2458-12-291

Miller, E., \& Sikes, H. D. (2015). Addressing Barriers to the Development and Adoption of Rapid Diagnostic Tests in Global Health Invited Review Article. 1-21. https://doi.org/10.5772/61114

Moonasar, D., Goga, A. E., Frean, J., Kruger, P., Maharaj, R., \& Chandramohan, D. (2009). S CIENTIFIC L ETTERS Primary health workers 'proficiency in using malaria rapid diagnostic tests in Limpopo Province. 99(11).

Mubi, M., Kakoko, D., Ngasala, B., Premji, Z., Peterson, S., Björkman, A., \& Mårtensson, A. (2013). Malaria diagnosis and treatment practices following introduction of rapid diagnostic tests in Kibaha District, Coast Region , Tanzania. 1-8. https://doi.org/10.1186/1475-2875-12-293

Mukanga, D., Tibenderana, J. K., Peterson, S., Pariyo, G. W., Kiguli, J., Waiswa, P., Babirye, R., Ojiambo, G., Kasasa, S., Pagnoni, F., \& Kallander, K. (2012). Access, acceptability and utilization of community health workers using diagnostics for case management of fever in Ugandan children: a cross-sectional study. 1-10.

NMCP. (2013). National Malaria Control Programme Ghana Malaria Programme Review. June, 24. https://www.ghanahealthservice.org/downloads/ghana_malaria_programme_review_final_report_june_2013.pdf 
Ochodo, E., Garner, P. A., \& Sinclair, D. (2016a). Achieving universal testing for malaria. February. https://doi.org/10.1136/bmj.i107

Ochodo, E., Garner, P., \& Sinclair, D. (2016b). argue Eleanor Ochodo and colleagues. 107(February), 1-6. https://doi.org/10.1136/bmj.i107

Pan, A. P. (2015). Malaria rapid diagnostic test as point-of-care test: study protocol for evaluating the VIKIA $₫$ Malaria. 1-8. https://doi.org/10.1186/s12936-015-0633-3

Rauf, A., Anto, B. P., Koffuor, G. A., Buabeng, K. O., \& Abdul-kabir, M. (2014). Introducing Malaria Rapid Diagnostic Tests (MRDTs ) at Registered Retail Pharmacies in Ghana: Practitioners 'Perspective. 4(8), 943-953.

Salomão, C. A., Sacarlal, J., Chilundo, B., \& Gudo, E. S. (2015). Prescription practices for malaria in Mozambique: poor adherence to the national protocols for malaria treatment in 22 public health facilities. Malaria Journal, 18. https://doi.org/10.1186/s12936-015-0996-5

Stresman, G., Kobayashi, T., Kamanga, A., Thuma, P. E., Mharakurwa, S., Moss, W. J., \& Shiff, C. (2012). Malaria research challenges in low prevalence settings. 2-5.

Zongo, S., Farquet, V., \& Ridde, V. (2016). A qualitative study of health professionals ' uptake and perceptions of malaria rapid diagnostic tests in Burkina Faso. Malaria Journal, 1-11. https://doi.org/10.1186/s12936-016$1241-6$ 